https://www.journal-imab-bg.org

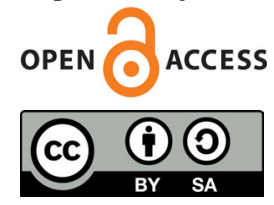

Original article

\title{
ANALYSIS OF POSTOPERATIVE PAIN AFTER EXTRACTION OF IMPACTED MANDIBULAR THIRD MOLARS AND ADMINISTRATION OF PREEMPTIVE ANALGESIA
}

\author{
Deyan Neychev ${ }^{1}$, Ivan Chenchev ${ }^{1}$, Kiril Simitchiev ${ }^{2}$ \\ 1) Department of Oral Surgery, Faculty of Dental Medicine, Medical University \\ -Plovdiv, Bulgaria \\ 2) Department of Analytical Chemistry and Computer Chemistry, University of \\ Plovdiv Paisii Hilendarski, Bulgaria.
}

\begin{abstract}
Objective: Postoperative pain control after extraction of impacted mandibular third molars is important for patients because of the effect of pain symptoms on the healing process and quality of life. The objective of this study is to make a quantitative and qualitative assessment of the postoperative pain in patients to whom preemptive analgesia was administered.

Material and Methods: This is a randomised, placebo-controlled study in 80 patients who underwent surgical removal of impacted mandibular third molars. The patients were divided into three groups - with the preemptive administration of placebo, metamizole sodium and nimesulide. The short form of the McGill Pain Questionnaire (SF-MPQ) and the Visual Analogue Scale (VAS) were used for assessment and analysis of postoperative pain.

Results: For the first 24 hours after molar extraction the comparison of the values of the various pain components showed a superior effect of the preemptive analgesia with nimesulide for the alleviation of sensory and total pain compared to metamizole sodium and placebo. In all three groups, pain intensity was highest at postoperative hour 6 .

Conclusion: Preemptive use of NSAIDs in the extraction of impacted mandibular third molars reduces the perception of postoperative pain and its intensity.
\end{abstract} MPQ,

Keywords: pain control, preemptive analgesia, SF-

\section{INTRODUCTION}

The extraction of a third molar is a routine surgical procedure. This type of surgical procedures is used as a pain model $[1,2]$ for the introduction of new analgesics and analgesic techniques in the postoperative period. The removal of impacted mandibular third molars is usually a planned surgical procedure, after which a moderate to severe pain occurs within 1 to 3 hours after the operation, requiring the use of analgesics $[3,4,5]$. The acute postoperative pain in the early postoperative period and the possibilities for minimising and controlling it are of scientific interest. The postoperative pain is a complicated response to the tissue injury resulting from the operation that stimulates central nervous system hypersensitivity. It may occur after any surgical procedure, whether it is minor oral surgery or another type of surgery. After surgical interventions, prolonged pain stimuli cause suffering, harm to the body and post-operative complications that may have a negative effect on patient recovery. This makes necessary to take into account pain characteristics and its intensity in order to assess the need for a particular type of analgesic intervention. Pain assessment can be done using different questionnaires and scales by which pain intensity is determined. In 1975, Melzack, based on earlier studies conducted collaboratively with Torgerson [6], developed the so-called McGill questionnaire. McGill Pain Questionnaire is one of the most commonly used tools for pain assessment, including its sensory and affective characteristics. Based on the original version, Melzack developed a short form of this questionnaire [7]. Completing it takes less time, and it is more informative because it was supplemented with a Visual Analogue Scale (VAS) and a Present Pain Intensity (PPI) scale. The short form of the McGill Pain Questionnaire (SF-MPQ) provides quantifiable information of the sensory and affective experience of the pain and enables mathematical calculations and comparisons. SF-MPQ is a useful tool in pain studies. There is a high degree of correlation of the quantitative characteristics of the pain between the scores obtained using the short form and the original form [7]. The credibility of both forms is documented, which justifies their use [8]. Notwithstanding all the above, the use of the short form of the McGill Pain Questionnaire depends on the patient as well - whether the patient is able to understand many of the listed verbal descriptors relating to pain. The defined inclusion criteria (including patient's intellectual level) are important for the scores of the questionnaires and of the scales used for pain assessment.

SF-MPQ cannot distinguish between different pain syndromes; it is unsuitable for differential diagnosis. Best results can be obtained using the short form of the ques- 
tionnaire while monitoring the changes in pain parameters over time and evaluating the outcome. According to Piotrowski [9], the short form of the McGill Questionnaire is one of the most commonly used tools for pain assessment, both in a clinical setting and in research studies.

One of the modern analgesic techniques in recent years is the use of preemptive analgesia. The use of preemptive analgesia in surgical procedures is based on the studies by Woolf [10] on central sensitization.

\section{OBJECTIVE OF THE STUDY}

To find the qualitative characteristics of postoperative pain after extraction of impacted mandibular third molars and administration of preemptive analgesia with nimesulide, metamizole sodium and placebo, using the short form of the McGill Pain Questionnaire.

\section{MATERIAL AND METHODS}

Participants in the study

This is a randomised, double-blind, placebo-controlled study in 80 patients who underwent surgical removal of impacted mandibular third molars. It was conducted at the Department of Oral Surgery of the Faculty of Dental Medicine at the Medical University of Plovdiv. The study was approved by the Ethics Committee of the Medical University of Plovdiv. Only patients who met all of the inclusion and none of the exclusion criteria were enrolled in the study. Inclusion criteria: Clinically healthy patients at the age of 17 to 45, with indications for extraction of a mandibular third molar; No evidence of pain related to the mandibular third molar to be extracted; Third molars to be extracted must be of Pell and Gregory class 1 and 2. Exclusion criteria: Patients at age outside the age range for the study; Pregnancy; Allergy to nimesulide, metamizole or to lactose - the main ingredient of the placebo; Acute inflammation in the area of the tooth to be extracted; Taking antibiotic preparations and nonsteroidal anti-inflammatory drugs (NSAIDs) in the last seven days; Patients needing antibiotic prophylaxis. The participation of each patient in this study was voluntary, and the patient was enrolled after obtaining written informed consent.

Study design

Following simple randomisation, patients were divided into three groups depending on which medication they received: 30 patients received nimesulide $(100 \mathrm{mg})$, 30 patients received metamizole sodium $(500 \mathrm{mg})$, and 20 patients received placebo. Thirty minutes prior to the extraction, each patient was given a numbered vessel_containing 10 tablets of one of the three pharmaceutical products._Each patient took 1 tablet 30 minutes before the operation. Then the drug was administered at every 12 hours.

Surgical technique

The surgical intervention was surgical removal of an impacted mandibular third molar. $4 \%$ solution of articaine hydrochloride with epinephrine concentration of 1:100000 was used for analgesia. The operations for surgical removal of an impacted mandibular third molar were performed us- ing a standard technique with access through a triangular mucoperiosteal flap.

\section{Assessment of postoperative pain}

The short form of the McGill Pain Questionnaire (SFMPQ) provides quantifiable information of the sensory and affective experience of the pain and enables mathematical calculations and comparisons. This questionnaire consists of 15 pain descriptors. Descriptors 1 to 11 represent the sensory component of pain, and descriptors 12 to 15 represent the affective component. Each descriptor is ranked on an intensity scale: 0 - no pain, 1 - mild pain, 2 - moderate pain, and 3 - severe pain. The Pain Rating Index (PRI), introduced in the standard form, is also used in the short form of the McGill Pain Questionnaire in order to differentiate the various pain components (PRI-S, PRI-A and PRI-T). Three pain values corresponding to the final scores of summation of the intensity values are obtained: for sensory, affective and total pain experience. The short form of the McGill Pain Questionnaire is accessible and easy to understand, and it takes about 5-10 minutes to record the patient's experience. The short form of the McGill Pain Questionnaire was completed at postoperative hour 2, 6, 12, 24, 48 and 72.

\section{Statistical analysis}

Non-parametric methods were used to perform the statistical analysis of sensory pain, affective pain, total pain and PPI since they are scalar values (natural numbers) for which the assumption of normal distribution of the values is not satisfied. For any fixed time moment the MannWhitney U and Kruskal-Wallis tests were used to detect statistical differences among the studied groups. A Wilcoxon signed-rank test and the Friedman test were used to evaluate the change in the scores in a particular group of patients as a function of time. The significance level was set at $5 \%$.

\section{RESULTS}

The allocation of the patients among the three investigated groups was statistically identical in respect to the subjects' gender and age.

For the first 24 hours after the molar extraction, the results of the analysis of the short form of the McGill Pain Questionnaire demonstrate a superior effect of the preemptive analgesia with nimesulide for the alleviation of sensory and total pain compared to metamizole sodium and placebo (Figure 1).

The effect of the analgesic intervention (preemptive administration) on the individual pain components was assessed as a function of time with the Friedman test (Figure 1). The highest increase in the scores of the pain components was observed in the placebo group. In the metamizole group, all pain components were influenced to some extent. However, a dominant suppression of the sensory and affective pain, as well as of the final (total) pain with the preemptive administration of nimesulide was proved. This was particularly pronounced during the first 24 hours of the early postoperative period. 
Fig. 1. Changes in sensory, affective and total pain after preemptive analgesia with nimesulide, metamizole sodium and placebo as a function of time

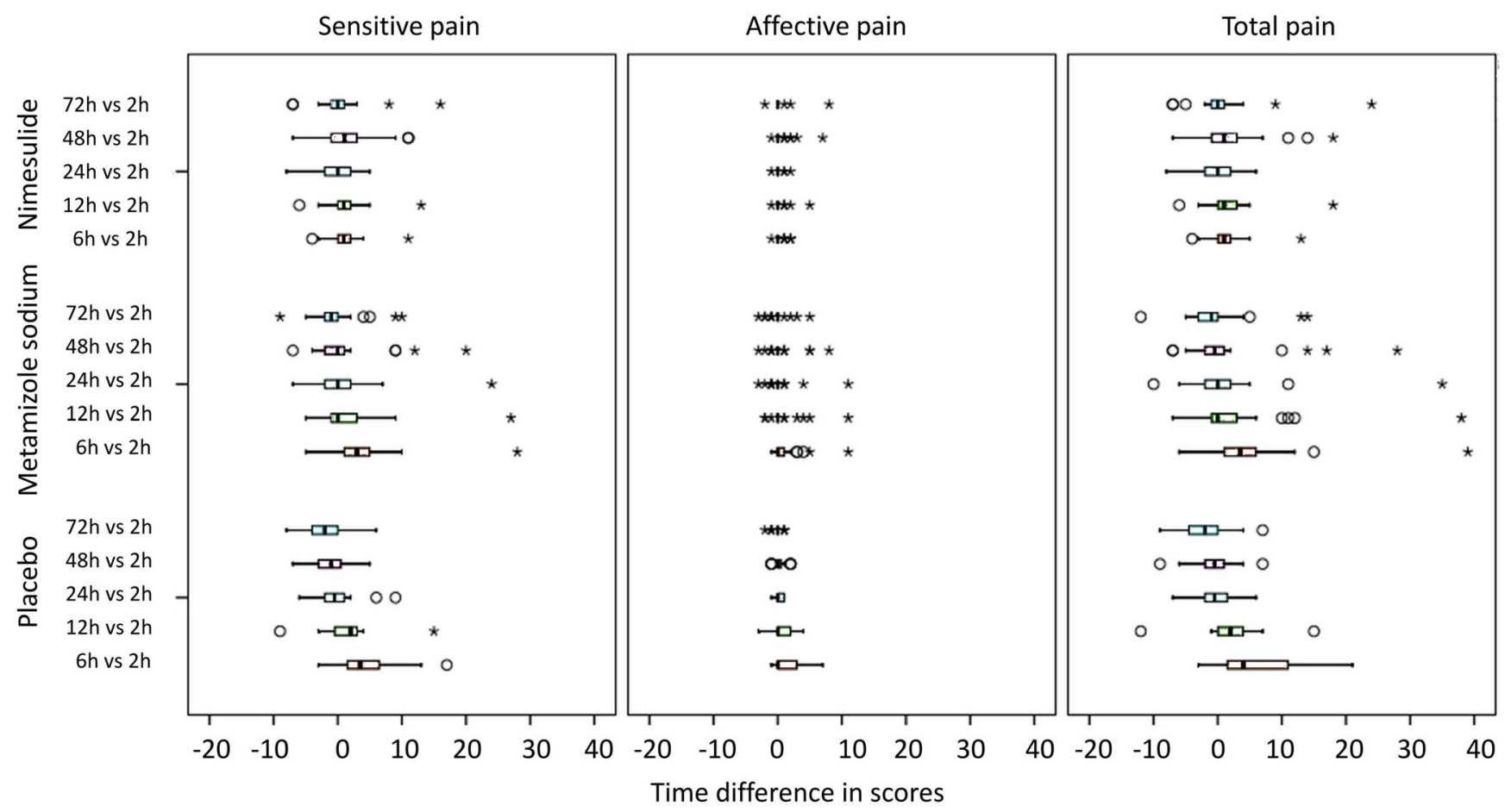

The scores of another component of McGill's questionnaire - VAS - show a great dissonance in the intensity of pain in the three treatment groups. In Table 1 are shown the dynamic changes in pain intensity after extraction of impacted mandibular third molars, again presented as a function of time. The data obtained for the comparison of the intensity in the three groups were statistically significant. The lowest intensity of postoperative pain was found in the nimesulide group.

Table 1. Changes in pain intensity based on VAS as a function of time

\begin{tabular}{|l|c|c|c|c|c|c|c|c|c|c|}
\hline \multirow{2}{*}{ Treatment } & \multicolumn{10}{|c|}{ Difference in VAS, mm } \\
\cline { 2 - 12 } & \multicolumn{2}{|c|}{$\mathbf{6 h}$ vs 2h } & \multicolumn{2}{|c|}{$\mathbf{1 2 h}$ vs 2h } & \multicolumn{2}{|c|}{$\mathbf{2 4 h}$ vs 2h } & \multicolumn{2}{|c|}{$\mathbf{4 8 h}$ vs 2h } & \multicolumn{2}{|c|}{$\mathbf{7 2 h}$ vs 2h } \\
\cline { 2 - 12 } & Mean & SD & Mean & SD & Mean & SD & Mean & SD & Mean & SD \\
\hline Placebo & 24,5 & 26,8 & 14,9 & 19,5 & 3,7 & 21,5 & $-0,9$ & 21,7 & $-7,6$ & 20,3 \\
\hline Metamizole sodium & 20,8 & 22 & 10,1 & 25,7 & 5,8 & 22,4 & 3,5 & 23 & $-1,3$ & 25,5 \\
\hline Nimesulide & 10,3 & 19,3 & 8,6 & 19,5 & 4,4 & 21,6 & 9,8 & 23,7 & 2,4 & 15,5 \\
\hline
\end{tabular}

The PPI scores were similar to VAS scores. Low-intensity pain over time was observed in patients treated with nimesulide. In the other two groups, the pain intensity was significantly higher and was most pronounced in the placebo group. 
Fig. 2. Changes in PPI after preemptive analgesia with nimesulide, metamizole sodium and placebo as a function of time

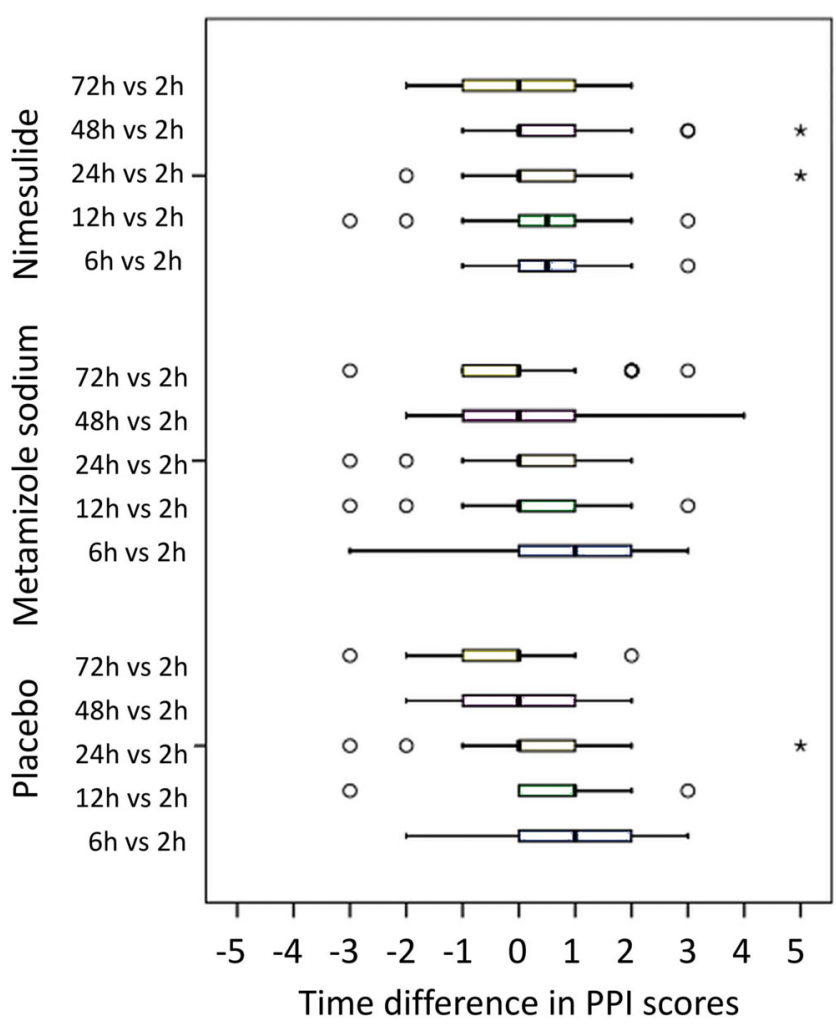

\section{DISCUSSION}

The issues related to achieving good postoperative analgesia are many and various - insufficient competence, fear of complications, poor knowledge of analgesic drugs, poor assessment of pain, etc. [11].

There are numerous techniques for analgesic treatment of postoperative pain, but the interest in recent years has been focused on the preemptive analgesia. This is due to the development of the fundamental sciences associated with the discovery of central sensitization by Woolf [10].

According to a number of authors, the combination of preemptive analgesia with local anaesthesia makes the control of postoperative pain more effective [12, 13].

Quantification of pain experience allows for adequate control of postoperative pain and assessment of the analgesic techniques used. According to the same authors, preemptive use of analgesia is appropriate in patients undergoing ambulatory surgery, together with administration of NSAIDs [14].

In their studies, some authors comment on the use of the short form of the McGill Pain Questionnaire and the need to exclude patients with cognitive problems from the study [15]. No such patients were enrolled in our study.

In order to evaluate the effectiveness of the preemptive analgesia, we used the SF-MPQ and compared patients' experience of pain at different time points. The results showed that there is no relationship between gender, age, and postoperative pain experience. These findings are in agreement with the findings of other authors [16].

Studies by some authors show that in surgical removal of impacted third molars, the sensory component of pain is leading [17]. The findings of our study are identical.

There are no unambiguous opinions on the quantitative assessment of pain in the extraction of impacted mandibular third molars and comparison of analgesic techniques - preemptive, postoperative, and placebo. Studies by some authors demonstrate that there is no significant difference between the study groups and postoperative pain assessment using SF-MPQ [18]. These results are completely contradictive to the results obtained by us.

One of the most commonly used tools for assessing pain intensity is VAS. It is easy to use and does not require serious preliminary preparation. The results of our study demonstrate much lower pain intensity in the group with preemptive administration of a NSAID (nimesulide) compared to the other two groups. Our findings are similar to those of identical placebo-controlled studies [19].

According to other authors, the preemptive administration of NSAIDs does result in a reduction in pain intensity assessed by VAS [20].

The PPI used in SF-MPQ showed that the scores are comparable to those of the visual analogue scale.

\section{CONCLUSION}

The use of preemptive analgesia with nimesulide results in significant reduction in the pain experience. Preemptive analgesia influences primarily the sensory component of pain, as well as the intensity of pain.

\section{List of abbreviations}

NSAID - Nonsteroidal anti-inflammatory drug PPI - Present Pain Intensity

PRI - Pain Rating Index

SF-MPQ - short form of the McGill Pain Questionnaire VAS - visual analogue acale.

\section{REFERENCES:}

1. Singla NK, Desjardins PJ, Chang PD. A comparison of the clinical and experimental characteristics of four acute surgical pain models: Dental extraction, bunionectomy, joint replacement, and soft tissue surgery Pain. 2014 Mar;155(3): 441-56. [PubMed]

\section{[CrossRef]}

2. Winter L Jr, Bass E, Recant B, Cahaly JF. Analgesic activity of ibuprofen in postoperative and surgical pain. Oral Surg. 1978 Feb;45(2): 159-66. [PubMed]

3. Barden J, Edwards JE, McQuay
HJ, Moore RA. Pain and analgesic response after third molar extraction and other postsurgical pain. Pain. 2004 Jan;107(1-2):86-90. [PubMed]

4. Cooper SA. Models for clinical assessment of oral analgesics. Am J Med. 1983 Nov;75(5A):24-9. 
[PubMed]

5. Tavares MG, Machado AP, Motta BG, Borsatto MC, Rosa AL, Xavier SP. Electro-acupuncture efficacy on pain control after mandibular third molar surgery. Braz Dent J. 2007 18(2): 15862. [CrossRef]

6. Melzack R, Torgerson WS. On the language of pain. Anesthesiology. 1971 Jan;34(1): 50-9. [PubMed]

7. Melzack R. The short-form McGill Pain Questionnaire. Pain. 1987 Aug;30(2):191-97. [PubMed]

8. Katz J, Melzack R. The McGill Pain Questionnaire: Development, psychometric properties, and usefulness of the long form, short form, and short form-2. In: Turk DC, Melzack R, editors. Handbook of pain assessment. 3. New York, NY: Guildford Press; 2011. pp. 45-66.

9. Piotrowski C. Review of the psychological literature on assessment instruments used with pain patients. $N A$ J Psychol. 2007; 9:303-306.

10. Woolf CJ. Central sensitization: Implications for the diagnosis and treatment of pain. Pain 2011 Mar; 152(3 Suppl): S2-S15. [PubMed] [CrossRef]

11. Ramsay MA. Acute postopera- tive pain management. Proc (Bayl Univ Med Cent). 2000 Jul;13(3):2447. [PubMed]

12. Vadivelu N, Mitra S, Schermer E, Kodumudi V, David Kaye A, Urman RD. Preventive analgesia for postoperative pain control: a broader concept - Local Reg Anesth. 2014 May 29;7:17-22. [PubMed] [CrossRef]

13. Kelly DJ, Ahmad M, Brull SJ. Preemptive analgesia II: recent advances and current trends. Can J Anaesth. 2001 Dec;48(11):1091-101. [PubMed] [CrossRef]

14. Garimella V, Cellini C. Postoperative Pain Control. Clin Colon Rectal Surg. 2013 Sep;26(3):191-96. [PubMed] [CrossRef]

15. Gupta A, Kaur K, Sharma S, Goyal S, Arora S, Murthy RS. Clinical aspects of acute post-operative pain management \& its assessment. $J$ Adv Pharm Technol Res. 2010 Apr; 1(2):97-108. [PubMed]

16. Niederhagen B, Braumann B, Dierke-Dzierzon C, Albrecht S. [Postoperative pain after interventions in the area of the mouth-jaw-face.] [in German] Mund Kiefer Gesichtschir. 1997 Jul;1(4):229-34. [PubMed] [CrossRef]
17. Silva LC, Santos TD, Santos JA, Maia MC, Mendonça CG. Articaine versus lidocaine for third molar surgery: A randomized clinical study. Med Oral Patol Oral Cir Bucal. 2012 Jan 1;17(1):e140-45. [PubMed]

18. Mojsa IM, Pokrowiecki R, Lipczynski K, Czerwonka D, Szczeklik K, Zaleska M. Effect of submucosal dexamethasone injection on postoperative pain, oedema, and trismus following mandibular third molar surgery: a prospective, randomized, double-blind clinical trial. Int J Oral Maxillofac Surg. 2017 Apr;46(4):52430. [PubMed] [CrossRef]

19. Shah R, Mahajan A, Shah N, Dadhania AP. Preemptive analgesia in third molar impaction surgery. Natl J Maxillofac Surg. 2012 Jul-Dec;3(2): 144-47. [PubMed] [CrossRef]

20. Isiordia-Espinoza MA, PozosGuillen A, Martinez-Rider R, PerezUrizar J. Comparison of the analgesic efficacy of oral ketorolac versus intramuscular tramadol after third molar surgery: A parallel, double-blind, randomized, placebo-controlled clinical trial. Med Oral Patol Oral Cir Bucal. 2016 Sep 1;21(5):e637-43. [PubMed]

Please cite this article as: Neychev D, Chenchev I, Simitchiev K. Analysis of postoperative pain after extraction of impacted mandibular third molars and administration of preemptive analgesia. J of IMAB. 2017 Jul-Sep;23(3):16971701. DOI: https://doi.org/10.5272/jimab.2017233.1697

Received: 14/05/2017; Published online: 27/09/2017

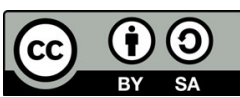

\author{
Corresponding author: \\ Deyan Z. Neychev, \\ Department of Oral Surgery, Faculty of Dental Medicine, \\ Medical University- Plovdiv; \\ 3, Hr. Botev str., 4000 Plovdiv, Bulgaria \\ E-mail: dneitchev@yahoo.com
}

